ARTICLE

\title{
https://doi.org/10.1038/s41467-021-24081-0
}

\section{Structural basis for the ARF GAP activity and specificity of the C9orf72 complex}

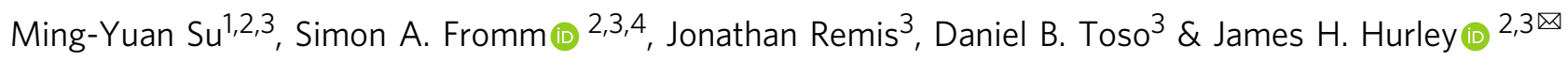

Mutation of C9ORF72 is the most common genetic cause of amyotrophic lateral sclerosis (ALS) and frontal temporal degeneration (FTD), which is attributed to both a gain and loss of function. C9orf72 forms a complex with SMCR8 and WDR41, which was reported to have GTPase activating protein activity toward ARF proteins, RAB8A, and RAB11A. We determined the cryo-EM structure of ARF1-GDP-BeF${ }_{3}^{-}$bound to C9orf72:SMCR8:WDR41. The SMCR8 longin and C9orf72longin domains form the binding pocket for ARF1. One face of the C9orf72longin domain holds ARF1 in place, while the SMCR8 ${ }^{\text {longin }}$ positions the catalytic finger Arg147 in the ARF1 active site. Mutations in interfacial residues of ARF1 and C9orf72 reduced or eliminated GAP activity. RAB8A GAP required $\sim 10$-fold higher concentrations of the C9orf72 complex than for ARF1. These data support a specific function for the C9orf72 complex as an ARF GAP. The structure also provides a model for the active forms of the longin domain GAPs of FLCN and NPRL2 that regulate the Rag GTPases of the mTORC1 pathway.

\footnotetext{
${ }^{1}$ School of Medicine, Southern University of Science and Technology, Shenzhen, China. ${ }^{2}$ Department of Molecular and Cell Biology, University of California, Berkeley, CA, USA. ${ }^{3}$ California Institute for Quantitative Biosciences, University of California, Berkeley, CA, USA. ${ }^{4}$ Present address: Imaging Centre, European

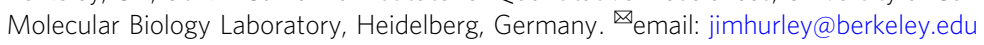


E xpansion of GGGGCC repeats in the first intron of C9ORF72 is the most prevalent genetic cause of two clinically overlapping neurodegenerative diseases, amyotrophic lateral sclerosis (ALS) and frontal temporal degeneration $(\mathrm{FTD})^{1,2}$. There are two mutually non-exclusive mechanisms for C9ORF72 expansion-induced pathogenesis in ALS/FTD, gain of function from the presence of toxic RNA and dipeptide repeats ${ }^{3,4}$, and loss of function due to reduced C9orf72 protein expression $^{5-7}$. The reduction or absence of C9orf72 has been proposed to alter vesicle trafficking ${ }^{8-10}$, autophagy ${ }^{10-13}$, lysosomal homeostasis ${ }^{14-16}$, actin dynamics ${ }^{17}$ and inflammation ${ }^{18}$. However, the direct biochemical function(s) of C9orf72 have been uncertain until recently.

C9orf72 exists in cells as a stable heterotrimer with Smith-Magenis chromosome regions 8 (SMCR8) and WD repeat-containing protein 41 (WDR41) 9,10,12-15. Both C9orf72 and its cellular binding partner SMCR8 are longin and DENN domain (differentially expressed in normal and neoplastic cells) containing proteins ${ }^{19}$. Two cryo-electron microscopy structural studies of the C9orf72:SMCR8:WDR41 complex found that SMCR8 is sandwiched between C9orf72 and WDR4120,21. Structural similarity was observed between C9orf72:SMCR8 and another double longin-DENN complex, the folliculin and folliculin-interacting protein 2 (FLCN-FNIP2) complex ${ }^{22,23}$. FLCN-FNIP2 is a GTPase activating protein (GAP) complex for RagC in the mTORC1 signaling pathway, with the putative catalytic Arg residue residing in the FLCN subunit. This suggested that C9orf72:SMCR8 might also be a GAP for some small GTPase $^{20,21}$. The C9orf72 complex was reported to have GAP activity for both ARF family proteins $s^{21}$, and the RAB proteins RAB8A and RAB11A ${ }^{20}$. The conserved Arg147 of SMCR8 was proposed to serve as the catalytic Arg finger typical of most GAP proteins. Mutation of SMCR8 Arg147 eliminated GAP activity, consistent with the Arg finger hypothesis. However, in the absence of a structure determination for the active state of any of these complexes, there is not yet a consensus in the field with respect to the Arg finger mechanism ${ }^{24}$.

The discovery that the C9orf72 complex has GAP activity against subsets of both ARF and RAB proteins was exciting in that both of these small GTPase families participate extensively in membrane traffic ${ }^{25}$, as does C9orf $72^{5,8,26}$. ARF6 and RAB11 vesicles ${ }^{27,28}$ have been implicated in retrograde vesicle transport in axons, such that excessive ARF6 GTP and RAB11GTP have been proposed to inhibit anterograde transport and axonal regeneration ${ }^{29}$. Nevertheless, the reported dual specificity of the C9orf72 complex is unusual. ARF and RAB proteins belong to distinct small GTPase families. Yet C9orf72 is inactive as a GAP against the close ARF relatives ARL8A and B, and also inactive against RAB1A and $7 \mathrm{~A}^{21}$. Thus, the C9orf72 complex is not simply a promiscuous GAP. In order to clarify the specificity of the C9orf72 complex GAP activity, we sought to determine structures of the complexes with representative ARF in GAPcompetent states. We selected ARF1 as a structurally wellcharacterized model for the ARF family, having previously found that GAP activity against ARF1, 5, and 6 was indistinguishable ${ }^{21}$. We found that the GAP reaction went to completion at an order of magnitude lower C9orf72 complex concentration for ARF1 as compared to RAB8A (greater than tenfold). Consistent with a preference for ARF proteins, it was possible to determine the structure of the ARF1:C9orf72:SMCR8:WDR41 complex in the presence of the GTP hydrolysis ground-state mimetic $\mathrm{BeF}_{3}{ }^{-}$. These data validate that the ARFs are specific substrates of C9orf72 complex GAP activity. The structure provides a model for the catalytic geometry of the larger longin-domain-based GAP family, including the FLCN:FNIP2 and GATOR1 complexes.

\section{Results}

We first characterized the concentration of C9orf72:SMCR8: WDR41 needed to produce complete hydrolysis of GTP by ARF1. An ARF1 construct with the N-terminal myristoylated amphipathic helix removed was used in all experiments, as this region was previously found to be unnecessary for its competence as a GAP substrate ${ }^{21}$. The concentration of ARF1 was fixed at $30 \mu \mathrm{M}$, and then treated with $0,0.3,0.6$, and $3-\mu \mathrm{M}$ C9orf72 complex for $15 \mathrm{~min}$ at $37^{\circ} \mathrm{C}$, and nucleotide products were monitored by high pressure liquid chromatogram (HPLC) analysis. The $0.3 \mu \mathrm{M}$ C9orf72:SMCR8:WDR41 sample, equivalent to a $1 / 100$ molar ratio relative to ARF1, was sufficient to completely hydrolyze all of the GTP-bound to ARF1 (Supplementary Fig. 1a).

To probe the structural basis of the catalytic mechanism, we attempted to assemble the GAP-ARF1 complex by mixing the GTP-locked mutant ARF1Q71L with the C9orf72:SMCR8:WDR41 protein complex. A stable complex was observed by gel-filtration chromatography (Supplementary Fig. 2a, b), but not by cryo-EM (Supplementary Fig. 2c). We inferred that the complex dissociated during vitrification. Following a precedent in which it was found necessary to use a fusion construct to trap the structure of the ASAP3:ARF6 GAP complex ${ }^{30}$, we fused the C terminus of ARF1 to the flexible $\mathrm{N}$-terminus of SMCR8, and coexpressed the fusion with C9orf72 and WDR41 in HEK293-GnTi cells as a chimeric C9orf72:ARF1-SMCR8:WDR41 complex (Fig. 1a). The N-terminal 50 residues of SMCR8 are disordered ${ }^{21}$, effectively serving as a flexible linker between ARF1 and SMCR8 of up to $150 \AA$ in length, which is similar to the longest axis of the C9orf72:SMCR8:WDR41 complex. The size exclusion profile demonstrated a similar yield and hydrodynamic behavior to the wild-type complex (Fig. 1b). HPLC analysis was used to assay the identity of the nucleotide bound in ARF1. Recombinant ARF1 protein as isolated from cells was $45-55 \%$ GTP-loaded, whereas the fusion version was only GDP-bound (Fig. 1c). This suggested to us that the fusion construct was catalytically active.

In order to capture a stable complex, we incubated the C9orf72: ARF1-SMCR8:WDR41 complex with both ground- or transitionstate analogs $\left(\mathrm{BeF}_{3}{ }^{-}\right.$and $\left.\mathrm{AlF}_{x}{ }^{-}\right)$and then tested if they could still promote the GTP hydrolysis for the purified ARF1. In the presence of $\mathrm{BeF}_{3}{ }^{-}$, but not $\mathrm{AlF}_{x}{ }^{-}, \mathrm{GAP}$ activity was inhibited. Thus, we generated a nucleotide hydrolysis ground-state analog ${ }^{31}$ by incubating the GDP-bound protein complex with $\mathrm{BeF}_{3}{ }^{-}$overnight, and visualized it by cryo-EM. A preliminary reconstruction of C9orf72:ARF1-SMCR8:WDR41 was obtained from a dataset collected on a $200-\mathrm{kV}$ Talos Arctica. This reconstruction showed extra density corresponding to ARF1 localized atop the two longin domains (Supplementary Fig. 3), and coordinates for the GTP-bound ARF1 model (PDB 1O3Y) ${ }^{32}$ fit the density. However, some regions were poorly resolved, and additional datasets were collected on a $300-\mathrm{kV}$ Titan Krios microscope (Supplementary Figs. 4-6). We were able to obtain a 3.9- $\AA$ cryo-EM reconstruction from an untilted dataset combined with $30^{\circ}$ and $40^{\circ}$ stage tilted datasets. It was possible to visualize the locations of the bound nucleotide and larger side-chains in this reconstruction, but smaller side-chains were not resolvable, nor were conformational details of larger side-chains.

The reconstruction showed that ARF1 sits in a $25 \AA$-wide concave pocket generated by the SMCR8 $8^{\text {longin }}$ and C9orf72longin domains. The ARF1 switch I and switch II regions, which are conformationally sensitive to the nucleotide state, directly contact the parallel helices of the SMCR ${ }^{\text {longin }}$-C9orf7 $2^{\text {longin }}$ dimer (Fig. 2a, b). The bowl formed by the two longin domains can be compared to the bowl of a spoon, and the parallel N-terminal helices of SMCR8 to the spoon handle. Relative to C9orf72, SMCR 8 longin has the more extensive interface with ARF1 (Fig. 2b). ARF1 is in its GTP-bound conformation, with respect to 
a
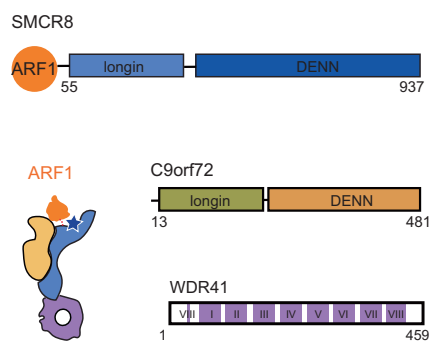

b

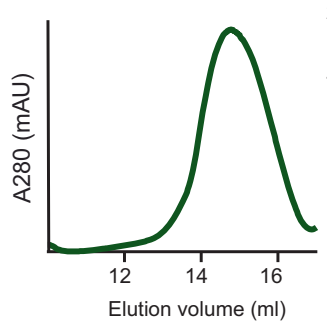

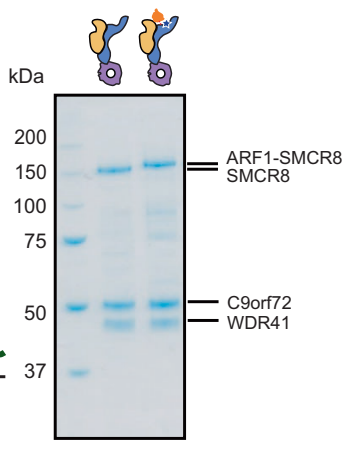

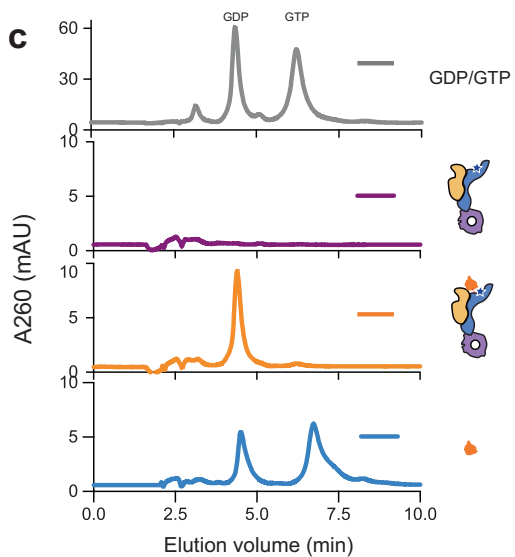

Fig. 1 Construct design of C9orf72:ARF1-SMCR8:WDR41 complex. a Diagram of the complex design. ARF1 is fused to the N-terminus of SMCR8 subunit. The blue star represents the position of the catalytic Arg residue of SMCR8. $\mathbf{b}$ Left, the Superose 6 size exclusion profile of the reconstituted ARF1-SMCR8: C9orf72:WDR41 complex. Right, the SDS-PAGE analysis of the peak fractions for ARF1-SMCR8:C9orf72:WDR41 complex compared with wild-type complex. c The HPLC analysis of the purified C9orf72:SMCR8:WDR41, C9orf72:ARF1-SMCR8:WDR41 complex, and ARF1. The elution profile for GDP/GTP is shown on the top panel.

all three of the regions whose conformation depends on the nucleotide state: the switch I, switch II, and interswitch regions (Fig. 2c).

SMCR8 Arg147 points directly toward the $\mathrm{BeF}_{3}{ }^{-}$, as predicted by the arginine finger model (Fig. 3a, b, left). The geometry is similar to that seen in the only other structure of a catalytically competent ARF GAP complex, that of ASAP3 and ARF6-GDP$\mathrm{AlF}_{x}$ (PDB: 3LVQ) ${ }^{30}$ (Fig. 3a, b, middle). In the ASAP3:ARF6structure, only one face of ARF6 contacts the GAP. In comparison, the C9orf72-SMCR8 longin spoon bowl cups ARF1 more extensively. ARF1 switch II sits on the parallel helices of the two longin domains and the loop of SMCR $8^{\text {longin, }}$, while switch I binds only to SMCR8 ${ }^{\text {longin. The }}$ more extensive cupping across the Switch I/II regions is seen in other GAP complexes, however, such as the TBC1D20:RAB1B-GDP- $\mathrm{BeF}_{3}{ }^{-}$complex (PDB: 4 HLQ $)^{33}$ (Fig. 3a, b, right).

ARF1 residues at the C9orf72 and SMCR8 interface were mutated and tested for GAP activity as monitored by HPLC (Fig. 4a, b). Mutation of residues (I49H, G50R) located at the switch I region largely reduced the activity compared to the ones at the switch II region (K73W), which is in consistent with the more extensive interface of SMCR8. We mutated the C9orf72 interface residues Trp33 and Asp34 in the double mutant W33G/ D34K (Fig. 4c). C9orf72 W33G/D34K:SMCR8:WDR41 complex was found to almost completely abolish GAP activity toward ARF1. We confirmed that C9orf72:SMCR8 was active toward RAB8A, albeit at much higher concentrations of the GAP complex than for ARF1. GAP activity with respect to RAB8A requires a $1 / 10$ molar ratio of C9orf72:SMCR8:WDR41 to hydrolyze the RAB8A proteins, at least approximately tenfold more than for ARF1 (Supplementary Fig. 1b). The I71R mutation of Ile71 on the C9orf72longin helix decreases the activity toward ARF1, whilst essentially abolishing activity toward RAB8A (Fig. 4).

\section{Discussion}

C9orf72-SMCR8 is one of a class of dimeric longin-DENN domain complexes that also include the FLCN-FNIP2 complex. Catalytic Arg residues of GATOR1 subunit NPRL2 ${ }^{34}, \mathrm{FLCN}^{22,23}$, and SMCR $8^{20,21}$ have been proposed to mediate GAP activity by an Arg finger mechanism ${ }^{35}$. We have now trapped and visualized the ARF1 GAP complex of C9orf72:SMCR8, confirming that the proposed Arg147 residue of SMCR8 serves as a catalytic Arg finger in the expected geometry. Thus far it has not been possible to trap GATOR1 or FLCN-FNIP2 in catalytically competent GAP complexes with their substrates RagA and RagC, owing to the transient nature of the complexes and competition with more stable inhibitory states. This has led to questions about whether the Rags might not in fact be regulated by the Arg fingers of FLCN and NPRL2, and might instead be more similar to the Ga subunit of heterotrimeric GTPases ${ }^{24}$. The structure provided here provides a basis for more precise modeling of the GATOR1 and FLCN GAP complexes in the active conformation (Supplementary Fig. 7). These models show that engagement of the Arg fingers of FLCN and NPRL2 in the FLCN-FNIP2 and GATOR1 complexes is sterically allowed and consistent with the mode of binding of SMCR8 to ARF1.

The great majority of the $>30$ known ARF GAP proteins contain a conserved ARF GAP domain, first described for ArfGAP1 ${ }^{36}$. Just a handful of ARF GAPs function without a canonical ARF GAP domain, including SEC23, the GAP for the ARF-related SAR1 GTPase, and the ELMO domain proteins ELMOD1- $3^{36}$. The data presented above define the C9orf72: SMCR8 longin-DENN complex as a new addition to the small set of protein folds possessing ARF GAP activity. We previously found that C9orf72:SMCR8 was active against ARF1, ARF5, and ARF6, although it had no activity against ARL8A and $\mathrm{B}^{21}$. The epitope immediately surrounding the GTP-binding site of ARFs 1-6 is highly conserved, and GAPs and GEFs are therefore typically active across the family ${ }^{37}$. ARF6 regulates axon extension ${ }^{38}$, and dominant-negative ARF6 expression rescues the axonal actin dynamics phenotype in C9ORF72 ALS patient cells $^{17}$. While ARF6 is the best candidate for a C9orf72 GAP substrate relevant to neurodegeneration, the structural features of the ARF1 complex presented here are expected to be essentially identical between the two.

Our data strongly support that C9orf72:SMCR8 is a GAP for ARF GTPases. Given the report that C9orf72:SMCR8 is also a GAP for RAB8A and RAB11 $A^{20}$, the question as to what are the physiological substrates of C9orf72:SMCR8 has become important in C9ORF72, ALS, and FTD research ${ }^{39,40}$. The data presented here affirm C9orf72:SMCR8 as a bona fide ARF GAP by presenting a well-ordered cryo-EM structure, which makes verifiable predictions of the activity of point mutants of both ARF1 and C9orf72. The generally much lower activity measured for RAB8A leaves the functional significance of the RAB GAP activity uncertain. It is possible that the presence of lipids or as-yet unidentified protein factors or post-translational modifications 

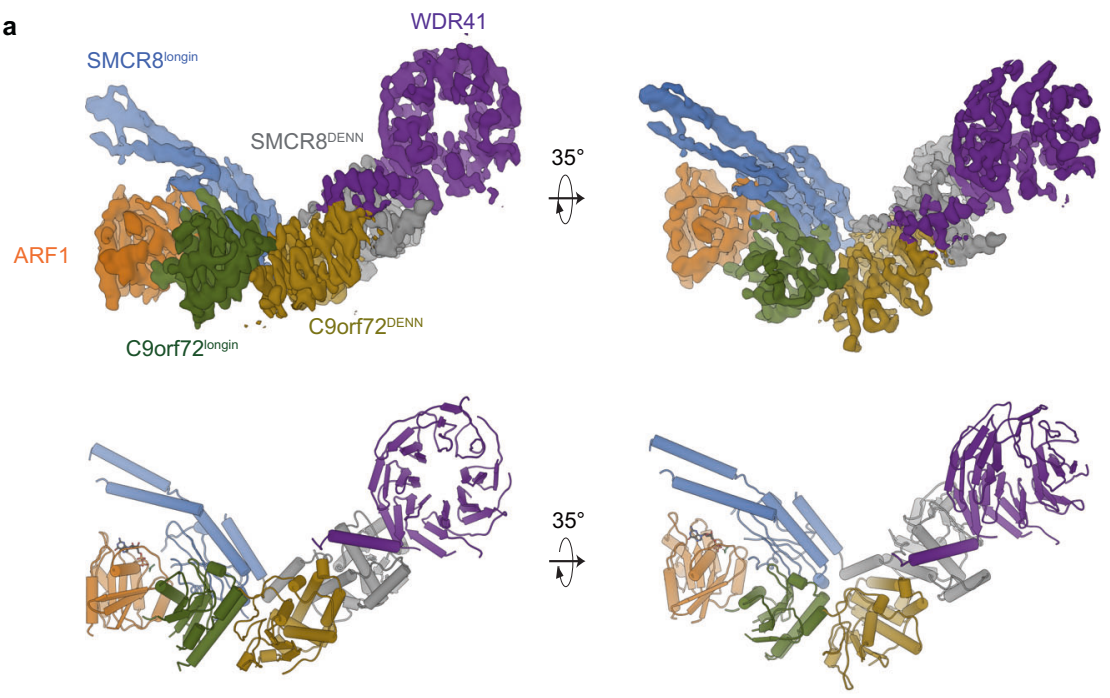

b

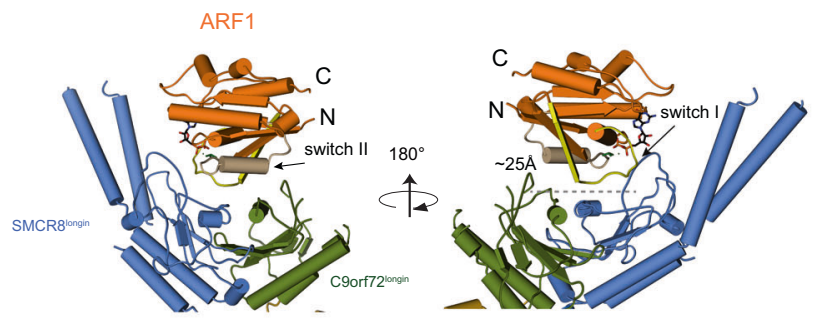

c

$$
\text { ARF1 }{ }^{\text {GDP }} \text { (PDB: 1RE0) }
$$$$
\text { ARF1 }^{\text {GTP }} \text { (PDB: 1O3Y) }
$$
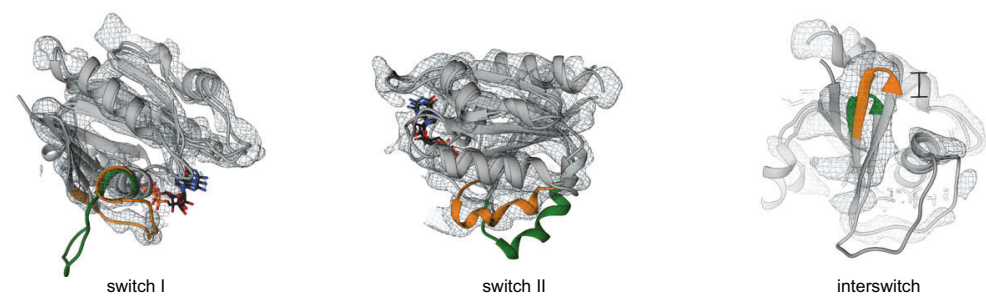

Fig. 2 Cryo-EM structure of C9orf72:ARF1-SMCR8:WDR41 complex. a Cryo-EM densities and the refined atomic model of the complex. The domain colored as followed: SMCR8 ${ }^{\text {longin }}$, cornflower blue; SMCR8 ${ }^{\text {DENN }}$, gray; C9orf72 ${ }^{\text {longin }}$, olive; C9orf72 ${ }^{\text {EENN }}$, goldenrod; WDR41, medium purple; ARF1: orange. b ARF1 sits on the top of the two longin domains. The switch I and II of ARF1 are colored in yellow and tan, respectively. c Structural comparison of ARF1 in GTP versus GDP-bound states (from the left to right panels, switch I and II and interswitch). ARF1 in GTP state fits into the density.

could increase the efficiency of RAB8A as a C9orf72:SMCR8 GAP substrate.

Another difference between the structures presented here and previously $^{21}$ is that our complex, expressed in HEK293 cells was monomeric, while material expressed in insect Sf9 cells was dimeric $^{20}$. We noticed a small population of C9orf72:SMCR8: WDR41 dimeric complex in our cryo-EM sample, and the dimeric complex was more populated when we cross-linked it at high concentrations of protein complex. This suggests that there is monomer-dimer equilibrium in the solution. Our observation of a catalytically competent monomeric complex does show that the monomer is the minimal entity capable of GAP activity.

The C9orf72 complex is reversibly localized to lysosomes in amino acid starvation through its WDR41 subunit ${ }^{41}$. WDR41 in turn binds to the lysosomal cationic amino acid transporter PQLC2 ${ }^{42}$. The PQLC2 binding site on WDR41 was recently identified $^{43}$, and is near the distal tip of the overall complex with respect to the ARF binding site. It is not apparent, based on existing structural data, how C9orf72:SMCR8:WDR41 could be localized to the lysosomal membrane to carry out GAP activity in cis on a small GTPase localized to the same membrane. There are few reports of ARFs localized to lysosomes, and thus would not be surprising that the complex is not designed to act in cis while lysosomally localized. This leaves two models for lysosomal regulation of ARF GAP activity. In the sequestration model, C9orf72 is active as a GAP in amino acid replete cells, but inactivated by lysosomal sequestration in starved cells. Alternatively, in the trans-activation model, the ARF GAP active site could project away from the lysosome to act on a pool of ARF localized to nearby membranes or cytoskeletal elements under starvation conditions.

The working hypothesis suggested by the data above is that C9orf72 haploinsufficiency in ALS and FTD results in an excess of ARFGTP in one or more localized pools within the cell. These might be in the vicinity of lysosomes and/or within axons. The locations and natures of these pools are major open questions whose answers call for further study. Given the numerous roles of ARF proteins in membrane traffic ${ }^{36}$, it would not be surprising if excess ARFGTP led to multiple defects in signaling, membrane traffic, and autophagy, which are correlates of C9ORF72 
a (this study)
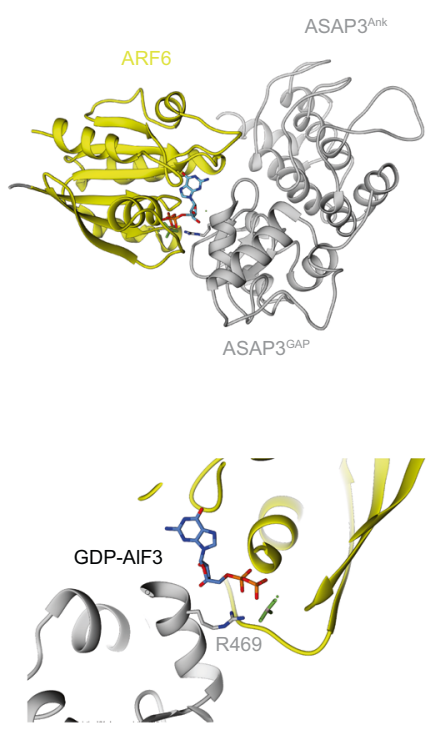

b

ARF1

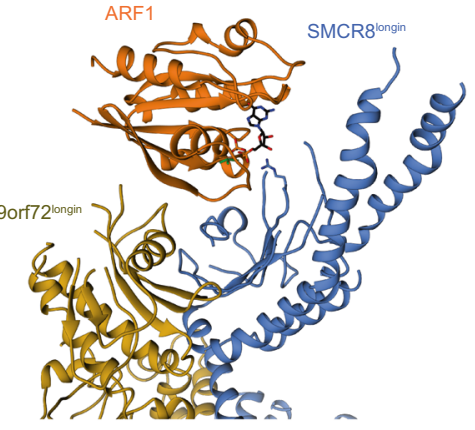

TBC1D20-Rab1B-GDP-BeF (PDB: 4HLQ)
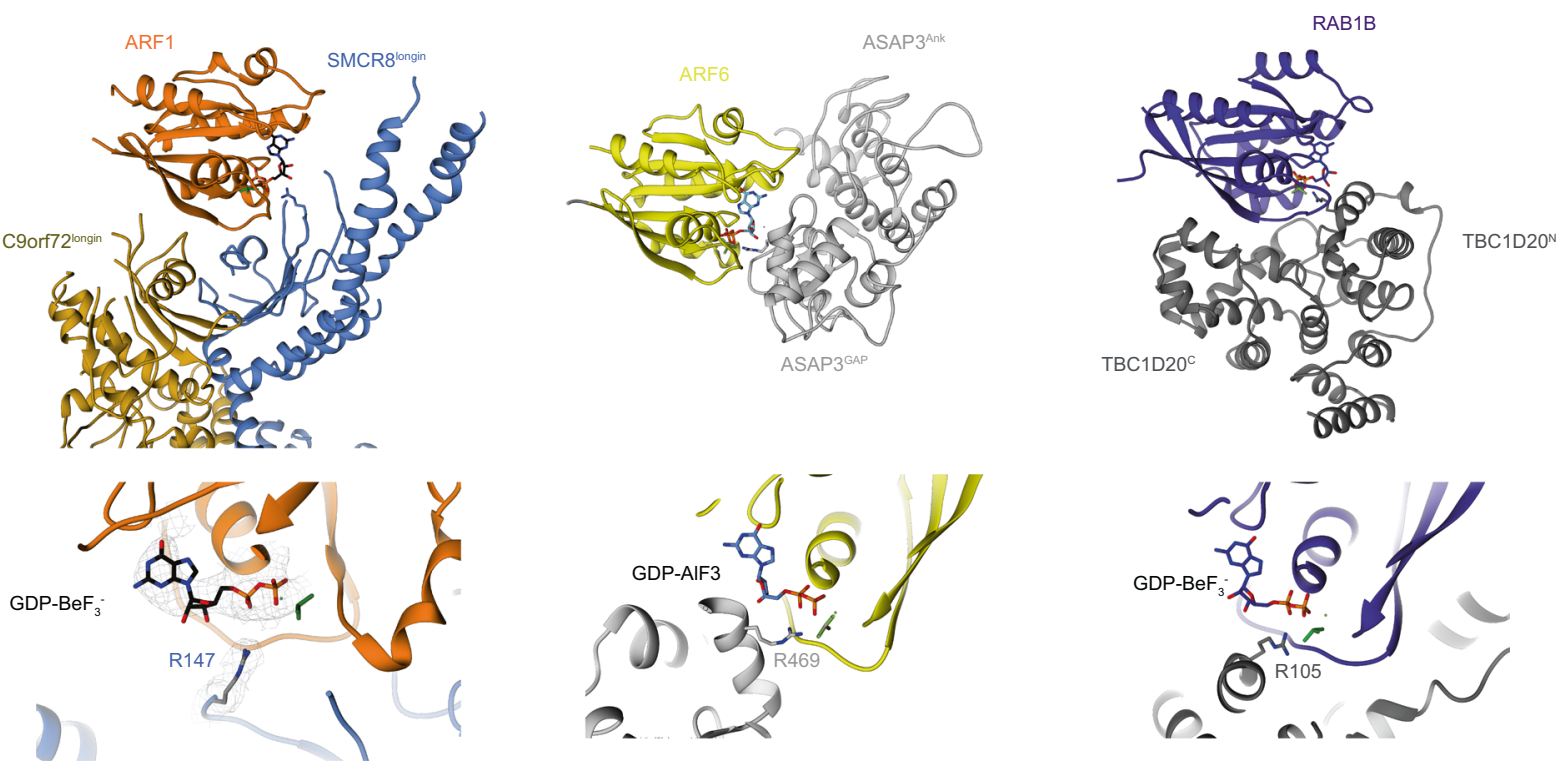

Fig. 3 Structure comparison between C9orf72:ARF1-SMCR8:WDR41 with other GAP:small GTPases. a Structure comparison between C9orf72:ARF1SMCR8:WDR41 (left) with ASAP3:ARF6 (middle) and TBC1D20:RAB1B (right). The small GTPases are shown in the same orientations. $\mathbf{b}$ Close-up views of the nucleotide-binding sites with catalytic Arginine finger residues pointing to the active sites.

haploinsufficiency in disease $\mathrm{C}^{6,7}$. This idea does suggest a potential therapeutic approach for ALS and FTD. In principle, ARFGTP levels could be rebalanced by inhibiting the ARF GEFs responsible for the GTP loading of the relevant local pools of ARFGTP, if the appropriate ARF GEF(s) were identified. This family of ARF activators comprises at least 15 members $^{36}$, which are the targets of the relatively unspecific natural product brefeldin A. A number of highly specific ARF GEF inhibitors have been identified, and strategies for their screening are well worked out ${ }^{44}$, lending support to the plausibility of such an approach.

\section{Methods}

Protein expression and purification. The gene encoding ARF1 (residues E17K181) was amplified and inserted into a pCAG vector encoding an N-terminal STREP-STREP-FLAG tagged SMCR8 using KpnI restriction sites (Supplementary Table 1), resulting in a STREP-STREP-FLAG-ARF1-SMCR8 construct. The pCAG vector encoding an N-terminal glutathione S-transferase (GST) followed by a TEV cleavage site was used for expression of C9orf72. WDR41 was cloned into a pCAG vector without a tag. The complex was expressed in HEK293-GnTi cells. The gene encoding RAB8A (residues M1-S181) was subcloned into pCAG resulting in a STREP-STREP-FLAG-RAB8A. All the ARF1 mutants were created by site-directed mutagenesis using KAPA HiFi HotStart ReadyMix. The C9orf72 mutants were generated by two-step PCR and ligated into the pCAG-GST vector.

HEK293-GnTi cells adapted for suspension were grown in Freestyle media supplemented with $1 \% \mathrm{FBS}$ and $1 \%$ antibiotic-antimycotic at $37^{\circ} \mathrm{C}, 80 \%$ humidity, $5 \% \mathrm{CO}_{2}$, and shaking at $140 \mathrm{rpm}$. Once the cultures reached $1.5-2$ million cells $\mathrm{mL}^{-1}$ in the desired volume, they were transfected as follows. For a 1-L transfection, 3-mL PEI (1 mg ml ${ }^{-1}, \mathrm{pH} 7.4$, Polysciences) was added to $50-\mathrm{mL}$ hybridoma media (Invitrogen) and $1 \mathrm{mg}$ of total DNA (isolated from transformed E. coli XL10-gold) in another 50-mL hybridoma media. Each $1 \mathrm{mg}$ of transfection DNA contained an equal mass ratio of the three C9orf72 complex subunit expression plasmids. PEI was added to the DNA, mixed, and incubated for $15 \mathrm{~min}$ at room temperature. One hundred milliliters of the transfection mix were then added to each 1-L culture. Cells were harvested after 3 days. Cells were lysed by gentle rocking in lysis buffer containing 50-mM HEPES, $\mathrm{pH} 7.4,200-\mathrm{mM} \mathrm{NaCl}, 2-\mathrm{mM} \mathrm{MgCl}_{2}, 1 \%$ (vol/vol) Triton X-100, 0.5-mM TCEP, protease inhibitors (AEBSF, Leupeptin, and Benzamidine) and supplemented with phosphatase inhibitors (50-mM NaF and 10$\mathrm{mM}$ beta-glycerophosphate) at $4^{\circ} \mathrm{C}$. Lysates were clarified by centrifugation $(15,000 \mathrm{~g}$ for $40 \mathrm{~min}$ at $4^{\circ} \mathrm{C}$ ) and incubated with 5-mL glutathione Sepharose $4 \mathrm{~B}(\mathrm{GE}$ Healthcare) for $1.5 \mathrm{~h}$ at $4^{\circ} \mathrm{C}$ with gentle shaking. The glutathione Sepharose $4 \mathrm{~B}$ matrix was applied to a gravity column, washed with $100-\mathrm{mL}$ wash buffer $(20-\mathrm{mM}$ HEPES, $\mathrm{pH} 7.4,200-\mathrm{mM} \mathrm{NaCl}, 2-\mathrm{mM} \mathrm{MgCl}_{2}$, and $0.5-\mathrm{mM}$ TCEP), and purified complexes were eluted with $40-\mathrm{mL}$ wash buffer containing $50 \mathrm{mM}$ reduced glutathione. Eluted complexes were treated with TEV protease at $4{ }^{\circ} \mathrm{C}$ overnight. TEV-treated complexes were purified to homogeneity by injection on Superose 6 Increased 10/300 GL (GE Healthcare) column that was pre-equilibrated in gelfiltration buffer (20-mM HEPES, $\mathrm{pH} 7.4,200-\mathrm{mM} \mathrm{NaCl}, 2-\mathrm{mM} \mathrm{MgCl}_{2}$, and $0.5-\mathrm{mM}$ TCEP). For long-term storage, fractions from the gel-filtration chromatography were snap-frozen in liquid nitrogen and kept at $-80^{\circ} \mathrm{C}$.

For expression of $\mathrm{His}_{6}$-tagged ARF1 (residues E17-K181) and all the ARF1 mutants, the plasmids were transformed into E. coli BL21 DE3 star cells and induced with 1-mM IPTG for $3 \mathrm{~h}$. The cells were lysed in 50-mM Tris- $\mathrm{HCl} \mathrm{pH} \mathrm{8.0,}$ $300-\mathrm{mM} \mathrm{NaCl}, 2-\mathrm{mM} \mathrm{MgCl}_{2}, 10-\mathrm{mM}$ imidazole, $0.5-\mathrm{mM}$ TCEP, and 1-mM PMSF by ultrasonication. The lysate was centrifuged at $15,000 \times g$ for $30 \mathrm{~min}$. The supernatant was loaded into Ni-NTA resin and washed with 20 and $50-\mathrm{mM}$ imidazole and eluted with $300-\mathrm{mM}$ imidazole. The eluate was further purified on a Superdex 75 10/300 GL (GE Healthcare) column equilibrated in 20-mM HEPES, $\mathrm{pH}$ 7.4, 200-mM NaCl, 2-mM MgCl , and 0.5-mM TCEP. STREP-STREP-FLAG tag RAB8A (residues M1-S181) was expressed in HEK293-GnTi cells and purified by Strep resin and eluted in $10-\mathrm{mM}$ desthiobiotin buffer. The eluted protein was applied on Superdex 75 10/300 GL column. The nucleotide-binding state of each small GTP-binding protein was assessed by HPLC (see below). The purified RAB8A was GDP-bound and our previous data demonstrated that it had spontaneous guanine exchange activity ${ }^{21}$, therefore RAB8A was incubated with GTP at room temperature for $2 \mathrm{~h}$ and applied to a PD 10 column to remove the excess nucleotide. The resulting RAB8A sample had roughly 45\% GTP-bound. $\mathrm{ARF}^{149 \mathrm{H} / \mathrm{G} 50 \mathrm{R}}$ and $\mathrm{ARF} 1^{149 \mathrm{H} / \mathrm{G} 50 \mathrm{R} / \mathrm{K} 73 \mathrm{~W}}$ were also GDP-bound, therefore the GTP reloading protocol was also applied to these samples.

For the assembly of C9orf72:SMCR8:WDR41 with ARF1Q71L complex, $15 \mu \mathrm{M}$ of C9orf72:SMCR8:WDR41 was incubated with 15-fold of ARF1 ${ }^{\text {Q71L }}$ in 500- $\mu 1$ overnight and injected into Superose 6 Increased 10/300 column.

The proteins used in this study are shown in Supplementary Fig. 8 and the uncropped gels are presented in Supplementary Fig 9.

Cryo-electron microscopy grid preparation and data imaging. For the Talos Arctica dataset, the purified complex was diluted to $0.16-0.2 \mathrm{mg} / \mathrm{ml}$ in $20-\mathrm{mM}$ HEPES pH 7.4, 200-mM NaCl, 2-mM $\mathrm{MgCl}_{2}$, and 0.5-mM TCEP, and incubated with $\mathrm{BeF}_{3}{ }^{-}$overnight. $3.5 \mu \mathrm{L}$ of sample was deposited onto freshly glow-discharged (PELCO easiGlow, $30 \mathrm{~s}$ in air at $20 \mathrm{~mA}$ and $0.4 \mathrm{mbar}$ ) C-flat $1.2 / 1.3-3 \mathrm{Au}$ grids (Electron Microscopy Sciences), and blotted for $4 \mathrm{~s}$ using a Vitrobot Mark IV (FEI) with 30-s incubation, blot force 8 and $100 \%$ humidity and temperature set to $22^{\circ} \mathrm{C}$. The complex was visualized in the Talos Arctica operated at an acceleration voltage of $200 \mathrm{kV}$, and equipped with a Gatan K3 Summit director electron detector in super-resolution counting mode at $36,000 \times$, corresponding to a pixel size of $0.57 \AA$. In total, 2579 movies were acquired with the defocus range between -1 and $-2.5 \mu \mathrm{m}$ with a $3 \times 3$ image shift pattern prior to stage movement. All movies consisted of 50 frames with a total dose of $50 \mathrm{e}^{-} / \AA^{2}$ and a total exposure time of $5.16 \mathrm{~s}$. 
a
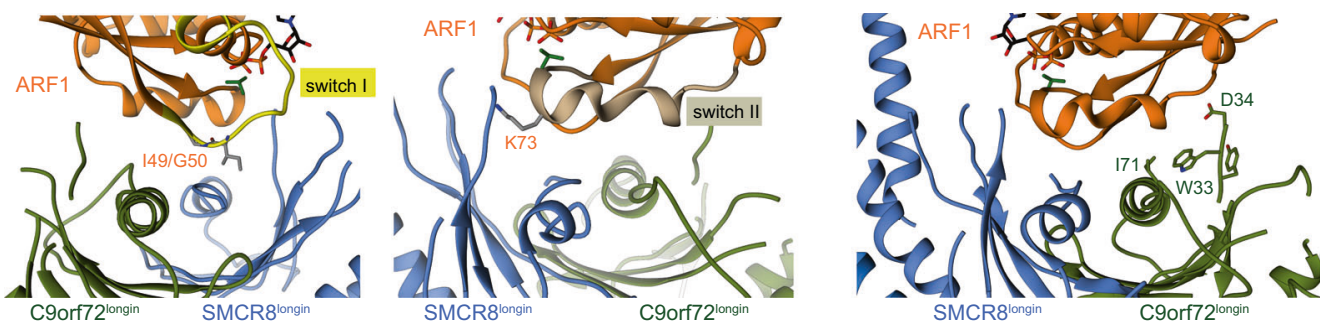

b

small GTPase only

treated with 1:100 C9orf72:SMCR8:WDR41
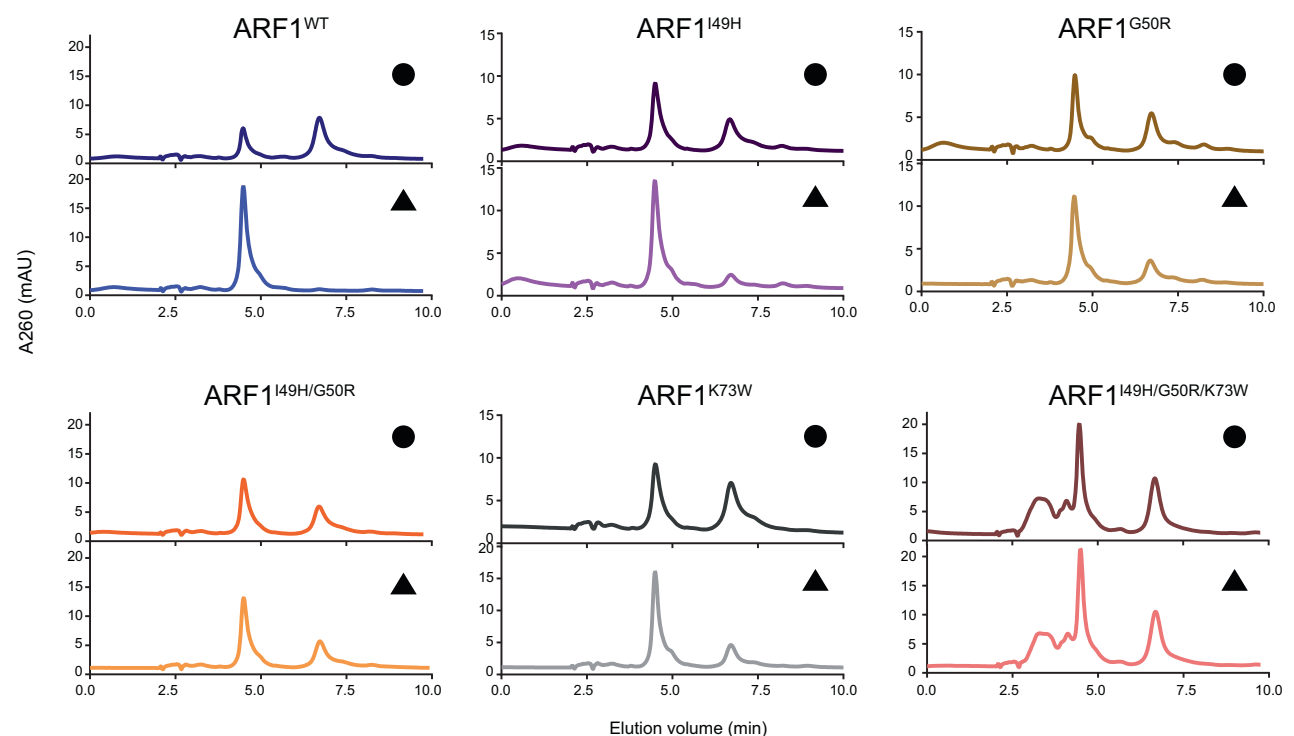

C

ARF1

RAB8A

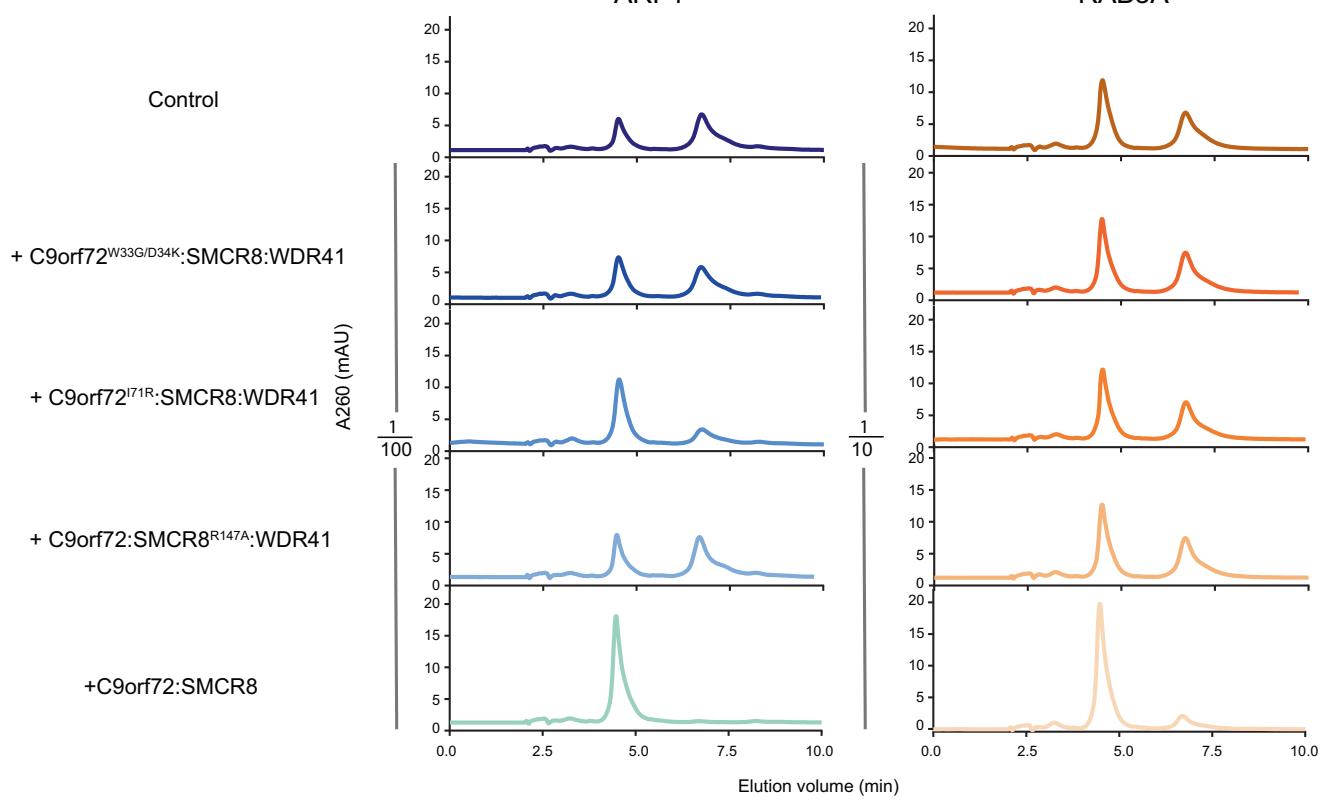

Fig. 4 Interfaces between ARF1 and C9orf72/SMCR8 and HPLC result of ARF1WT/mutants with C9orf72WT/mutants:SMCR8:WDR41. a Close-up views of the interfaces between switch I/switch II regions of ARF1 with C9orf72:SMCR8. b HPLC analysis of ARF1WT/mutants incubated with C9orf72:SMCR8: WDR41. The top panels are the control reactions for each ARF1 proteins, either wild-type or mutants. The bottom ones are incubated with 1/100 molar ratio of C9orf72:SMCR8:WDR41. c ARF1 or RAB8A incubated with 1/100 or 1/10 molar ratio of C9orf72WT/mutants:SMCR8:WDR41, respectively. The experiments in $\mathbf{b}$, c are carried out at least three times, and one representative plot is shown. 
For the untilted Titan Krios dataset, grids were prepared in the same manner and the complex was collected using a Titan Krios G2 electron microscope (FEI) operating at $300 \mathrm{kV}$ with a Gatan Quantum energy filter (operated at $20-\mathrm{eV}$ slit width) using a K3 summit direct electron detector (Gatan, Inc.) in super-resolution counting mode at magnification $81,000 \times$, corresponding to a pixel size of $0.47 \AA$ on the specimen level. In total, 10,188 movies were collected with defocus of -1.2 to $-2.5 \mu \mathrm{m}$ with a $3 \times 3$ image shift pattern prior to stage movement. Movies consisted of 50 frames, with a total dose of $50 \mathrm{e}^{-} / \AA^{2}$ and a total exposure time of $2.929 \mathrm{~s}$.

The Titan Krios dataset with a stage tilt of $30^{\circ}$ was obtained using $4-\mu \mathrm{L}$ sample applied on freshly glow-discharged (PELCO easiGlow, $25 \mathrm{~s}$ in air at $25 \mathrm{~mA}$ and 0.4 mbar) UltrAufoil R1.2/1.3 grid (Ted Pella) and plunged into liquid ethane after 30$\mathrm{s}$ incubation and blotting for $4 \mathrm{~s}$ using a Vitrobot Mark IV (FEI) with blot force 0 and $100 \%$ humidity and temperature set to $5{ }^{\circ} \mathrm{C}$. Four thousand five hundred and eighty-five movies were collected using SerialEM at around $-1.5-2.8-\mu \mathrm{m}$ defocus with $1 \times 3$ image shift pattern. The movies were exposed to a total dose of $50 \mathrm{e}^{-} / \AA^{2}$ for $5.326 \mathrm{~s}$ at CDS mode.

The Titan Krios dataset with a stage tilt of $40^{\circ}$ was collected using $4 \mu \mathrm{L}$ sample applied on freshly glow-discharged (PELCO easiGlow, $25 \mathrm{~s}$ in air at $25 \mathrm{~mA}$ and 0.4 mbar) GF-1.2/1.3-3Au-45nm-50 grid (Electron Microscopy Sciences) and plunged into liquid ethane after 30-s incubation and blotting for $3 \mathrm{~s}$ using a Vitrobot Mark IV (FEI) with blot force 8 and $100 \%$ humidity and temperature set to $4^{\circ} \mathrm{C}$. Six thousand one hundred and seventy-eight movies were collected using SerialEM at $-1.5-2.8-\mu \mathrm{m}$ defocus with $1 \times 3$ image shift pattern. The movies were exposed to a total dose of $50 \mathrm{e}^{-} / \AA^{2}$ for $5.326 \mathrm{~s}$ at CDS mode.

All of the data were acquired with SerialEM using custom macros for automated single particle data acquisition. Imaging parameters for the dataset are summarized in Supplementary Table 2.

Cryo-electron microscopy data processing. For the Talos Arctica dataset, drift and beam-induced motion correction was applied on the super-resolution movie stacks using MotionCor $2^{45}$ and using $7 \times 5$ patches and binned $2 \mathrm{x}$ by Fourier cropping to a calibrated pixel size of $1.14 \AA$ / pixel. The defocus values were estimated by CTFFIND $4^{46}$ from summed imaged without dose weighting. After manual check to remove empty or crystalline ice micrographs, 251 micrographs were discarded and the remaining 2328 micrographs were subjected to reference-free particle picking using gautomatch-v0.53 (http://www.mrc-lmb.cam.ac.uk/kzhang/ Gautomatch/). About four million particles were extracted with binning $4 \mathrm{x}$ in Relion $3.1^{47}$ and applied to iterations of three-dimensional (3D) classifications. The best class was selected and re-extracted with binning $1 \mathrm{x}$. The particles at the edges were removed and imported into cryoSPARC $\mathrm{v} 2{ }^{48}$ for further two-dimensional (2D) classification clean up and non-uniform refinement. The resulting 80,569 particles were refined to $4.84 \AA$ and used as reference map for further Titan Krios dataset processing. The workflow is depicted in Supplementary Fig. 3.

For the untilted Krios dataset, drift and beam-induced motion correction was applied on the super-resolution movie stacks using MotionCor 2 using $7 \times 5$ patches and binned $2 \mathrm{x}$ to a calibrated pixel size of $0.94 \AA /$ pixel. The defocus values were estimated by CTFFIND4 from summed imaged without dose weighting. Other processing procedures were performed within Relion 3.1. Seven thousand three hundred and forty-seven micrographs with CtfMaxResolution values better than 5 were selected for further processing. Around six millions of particles were picked using gautomatch-v0.53 (http://www.mrc-lmb.cam.ac.uk/kzhang/Gautomatch/) and then extracted with binning $6 \mathrm{x}$. The particles were subjected to 3D classification using a $45-\AA$ low-pass filtered reference generated by the reconstruction collected in Talos Arctica microscope. 888,231 particles from the best class were selected and refined to $7.755 \AA$. The refined particles were further cleaned up with rounds of $2 \mathrm{D}$ and $3 \mathrm{D}$ classification. The extracted, unbinned particles was refined to $4.5 \AA$ and applied to CTF refinement for beam-tilt and perparticle defocus determination as well as Bayesian polishing. The shiny particles were further refined to $3.7 \AA$. Map sharpening was performed with DeepEMhancer ${ }^{49}$ using the "tightTarget" or "highRes" models. The workflow is depicted in Supplementary Fig. 4.

For the Krios dataset with a stage tilt of $30^{\circ}$, data processing followed a similar pipeline of $0^{\circ}$ tilt dataset for processing except that first run a full-micrographbased CTF estimation, then pick particles using gautomatch and then subjected the particle coordinates to rerun local CTF estimation on a per-particle based in gctf $\mathrm{f}^{50}$ (Supplementary Fig. 4). For the Krios dataset with a stage tilt of $40^{\circ}$, the data were imported into cryoSPARC v2 for patch motion correction and patch CTF estimation. Particle picking was done using $2 \mathrm{D}$ class averages from $0^{\circ}$ tilt as reference for Autopick in Relion 3.1 and subjected to several rounds of 3D classification. The best classes were imported into cryoSPARC v2 for further rounds of 2D classification. The clean particles were applied to both global and local CTF refinement for then imported into Relion 3.1 to merge with other two datasets (Supplementary Fig. 4).

All reported resolutions are based on the gold-standard FSC 0.143 criterion. cryoSPARC v2 database files were converted to Relion star file using the UCSF PyEM package script (https://github.com/asarnow/pyem/). Particle orientation distributions were plotted using scripts (https://github.com/fuzikt/starpy). 3DFSC curve for the reconstruction was calculated on the 3 DFSC processing server ${ }^{51}$
Atomic model building and refinement. For the C9orf72:ARF1-SMCR8: WDR41 model, one copy of the coordinates from all three subunits (PDB 6V4U or 6LT0) ${ }^{20,21}$, WDR41 (PDB 6WHH), and mouse ARF1 (PDB: 1O3Y) ${ }^{32}$ were rigid body fitted separately into the density map using UCSF Chimera ${ }^{52}$. The Leu71 of ARF1 was mutated to its wild-type residue Gln and the GTP was replaced as GDP$\mathrm{BeF}_{3}{ }^{-}$. Atomic coordinates were refined by iteratively performing Phenix realspace refinement and manual building and correction of the refined coordinates in Coot. To avoid overfitting, the map weight was set to 1 and secondary structure restraints were applied during automated real-space refinement. Model quality was assessed using MolProbity ${ }^{53}$ and the map-vs-model FSC (Supplementary Table 2 and Supplementary Fig. 6b). A half-map cross-validation test showed no indication of overfitting (Supplementary Fig. 6a). Figures were prepared using UCSF Chimera. The cryo-EM density map has been deposited in the Electron Microscopy Data Bank under accession code EMD-23827 and the coordinates have been deposited in the Protein Data Bank under accession number 7MGE.

\section{High-performance liquid chromatography analysis of nucleotides identity} bound to small GTPases. The nucleotides bound to small GTPases were assessed by denaturing $30 \mu \mathrm{l}$ of protein to $95^{\circ} \mathrm{C}$ for $5 \mathrm{~min}$ followed by 5 -min centrifugation at $16,000 \times g$. The supernatant was loaded onto a HPLC column (Eclipse XDB-C18, Agilent). Nucleotides were eluted with HPLC buffer (10-mM tetra-nbutylammonium bromide, $100-\mathrm{mM}$ potassium phosphate $\mathrm{pH} 6.5,7.5 \%$ acetonitrile) at flow rate $0.6 \mathrm{ml} / \mathrm{min}$. The identity of the nucleotides was compared to GDP and GTP standards.

HPLC-based GAP assay. HPLC-based GTPase assays were carried out by incubating $30 \mu \mathrm{l}$ of $30-\mu \mathrm{M}$ GTPases with or without GAP complex at a 1:100, 1:50, or 1:10 molar ratio as indicated for $15 \mathrm{~min}$ at $37^{\circ} \mathrm{C}$. Samples were immediately boiled for at least $5 \mathrm{~min}$ at $95^{\circ} \mathrm{C}$ and centrifuged for $5 \mathrm{~min}$ at $16,000 \mathrm{~g}$. The supernatant was injected onto an HPLC column as described above. The experiments are carried out at least three times and one representative plot is shown.

Reporting summary. Further information on research design is available in the Nature Research Reporting Summary linked to this article.

\section{Data availability}

The data that support this study are available from the corresponding author upon reasonable request. EM density map has been deposited in the EMDB with accession number EMD-23827. Atomic coordinates have been deposited in the PDB with accession number $7 \mathrm{MGE}$

Received: 17 May 2021; Accepted: 27 May 2021;

Published online: 18 June 2021

\section{References}

1. DeJesus-Hernandez, M. et al. Expanded GGGGCC hexanucleotide repeat in noncoding region of C9ORF72 causes chromosome 9p-linked FTD and ALS Neuron 72, 245-256 (2011)

2. Renton, A. E. et al. A hexanucleotide repeat expansion in C9ORF72 is the cause of chromosome 9p21-linked ALS-FTD. Neuron 72, 257-268 (2011)

3. Lee, K. H. et al. C9orf72 dipeptide repeats impair the assembly, dynamics, and function of membrane-less organelles. Cell 167, 774-788.e17 (2016).

4. Guo, Q. et al. In situ structure of neuronal C9orf72 poly-GA aggregates reveals proteasome recruitment. Cell 172, 696-705.e12 (2018).

5. Shi, Y. et al. Haploinsufficiency leads to neurodegeneration in C9ORF72 ALS/ FTD human induced motor neurons. Nat. Med. 24, 313-325 (2018).

6. Boivin, M. et al. Reduced autophagy upon C9ORF72 loss synergizes with dipeptide repeat protein toxicity in $\mathrm{G} 4 \mathrm{C} 2$ repeat expansion disorders. EMBO J. 39, e100574 (2020)

7. Zhu, Q. et al. Reduced C9ORF72 function exacerbates gain of toxicity from ALS/FTD-causing repeat expansion in C9orf72. Nat. Neurosci. 23, 615-624 (2020).

8. Farg, M. A. et al. C9ORF72, implicated in amytrophic lateral sclerosis and frontotemporal dementia, regulates endosomal trafficking. Hum. Mol. Genet. 23, 3579-3595 (2014).

9. Sellier, C. et al. Loss of C9ORF72 impairs autophagy and synergizes with polyQ Ataxin-2 to induce motor neuron dysfunction and cell death. EMBO J. 35, 1276-1297 (2016)

10. Yang, M. et al. A C9ORF72/SMCR8-containing complex regulates ULK1 and plays a dual role in autophagy. Sci. Adv. 2, e1601167 (2016).

11. Webster, C. P. et al. The C9orf72 protein interacts with Rabla and the ULK1 complex to regulate initiation of autophagy. EMBO J. 35, 1656-1676 (2016). 
12. Sullivan, P. M. et al. The ALS/FTLD associated protein C9orf72 associates with SMCR8 and WDR41 to regulate the autophagy-lysosome pathway. Acta Neuropathol. Commun. 4, 51 (2016).

13. Jung, J. \& Behrends, C. Multifaceted role of SMCR8 as autophagy regulator. Small GTPases 11, 1-9 (2017).

14. Amick, J., Roczniak-Ferguson, A. \& Ferguson, S. M. C9orf72 binds SMCR8, localizes to lysosomes, and regulates mTORC1 signaling. Mol. Biol. Cell 27, 3040-3051 (2016).

15. Ugolino, J. et al. Loss of C9orf72 enhances autophagic activity via deregulated mTOR and TFEB signaling. PLoS Genet. 12, e1006443 (2016).

16. Lan, Y., Sullivan, P. M. \& Hu, F. SMCR8 negatively regulates AKT and MTORC1 signaling to modulate lysosome biogenesis and tissue homeostasis. Autophagy 15, 871-885 (2019).

17. Sivadasan, R. et al. C9ORF72 interaction with cofilin modulates actin dynamics in motor neurons. Nat. Neurosci. 19, 1610-1618 (2016).

18. McCauley, M. E. et al. C9orf72 in myeloid cells suppresses STING-induced inflammation. Nature 585, 96-101 (2020).

19. Zhang, D., Iyer, L. M., He, F. \& Aravind, L. Discovery of novel DENN proteins: implications for the evolution of eukaryotic intracellular membrane structures and human disease. Front. Genet. 3, 283 (2012).

20. Tang, D. et al. Cryo-EM structure of C9ORF72-SMCR8-WDR41 reveals the role as a GAP for Rab8a and Rab11a. Proc. Natl Acad. Sci. U. S. A. 117, 9876-9883 (2020).

21. Su, M. Y., Fromm, S. A., Zoncu, R. \& Hurley, J. H. Structure of the C9orf72 ARF GAP complex that is haploinsufficient in ALS and FTD. Nature 585, 251-255 (2020).

22. Lawrence, R. E. et al. Structural mechanism of a Rag GTPase activation checkpoint by the lysosomal folliculin complex. Science 366, 971-977 (2019).

23. Shen, K. et al. Cryo-EM structure of the human FLCN-FNIP2-Rag-ragulator complex. Cell 179, 1319-1329.e8 (2019).

24. Nawrotek, A. \& Cherfils, J. [New structures of mTORC1: focus on Rag GTPases]. Med Sci. 37, 372-378 (2021).

25. Dutta, D. \& Donaldson, J. G. Rab and Arf G proteins in endosomal trafficking. Methods Cell Biol. 130, 127-138 (2015).

26. Corrionero, A. \& Horvitz, H. R. A C9orf72 ALS/FTD ortholog acts in endolysosomal degradation and lysosomal homeostasis. Curr. Biol. 28, 1522-1535.e5 (2018).

27. Shiba, T. et al. Structural basis for Rab11-dependent membrane recruitment of a family of Rab11-interacting protein 3 (FIP3)/Arfophilin-1. Proc. Natl Acad. Sci. U. S. A. 103, 15416-15421 (2006).

28. Fielding, A. B. et al. Rab11-FIP3 and FIP4 interact with Arf6 and the exocyst to control membrane traffic in cytokinesis. EMBO J. 24, 3389-3399 (2005)

29. Nieuwenhuis, B. \& Eva, R. ARF6 and Rab11 as intrinsic regulators of axon regeneration. Small GTPases 11, 392-401 (2020).

30. Ismail, S. A., Vetter, I. R., Sot, B. \& Wittinghofer, A. The structure of an ArfArfGAP complex reveals a Ca2+ regulatory mechanism. Cell 141, 812-821 (2010).

31. Fisher, A. J. et al. X-ray structures of the myosin motor domain of Dictyostelium discoideum complexed with MgADP.BeFx and MgADP.AlF4. Biochemistry 34, 8960-8972 (1995).

32. Shiba, T. et al. Molecular mechanism of membrane recruitment of GGA by ARF in lysosomal protein transport. Nat. Struct. Biol. 10, 386-393 (2003).

33. Gavriljuk, K. et al. Catalytic mechanism of a mammalian Rab-RabGAP complex in atomic detail. Proc. Natl Acad. Sci. U. S. A. 109, 21348-21353 (2012).

34. Shen, K., Valenstein, M. L., Gu, X. \& Sabatini, D. M. Arg78 of Nprl2 catalyzes GATOR1-stimulated GTP hydrolysis by the Rag GTPases. J. Biol. Chem. 294, 2970-2975 (2019).

35. Ahmadian, M. R., Stege, P., Scheffzek, K. \& Wittinghofer, A. Confirmation of the arginine-finger hypothesis for the GAP-stimulated GTP-hydrolysis reaction of Ras. Nat. Struct. Biol. 4, 686-689 (1997).

36. Sztul, E. et al. ARF GTPases and their GEFs and GAPs: concepts and challenges. Mol. Biol. Cell 30, 1249-1271 (2019).

37. Cherfils, J. Arf GTPases and their effectors: assembling multivalent membrane-binding platforms. Curr. Opin. Struct. Biol. 29, 67-76 (2014).

38. Hernández-Deviez, D. J., Roth, M. G., Casanova, J. E. \& Wilson, J. M. ARNO and ARF6 regulate axonal elongation and branching through downstream activation of phosphatidylinositol 4-phosphate 5-kinase alpha. Mol. Biol. Cell 15, 111-120 (2004).

39. Pang, W. \& Hu, F. Cellular and physiological functions of C9ORF72 and implications for ALS/FTD. J. Neurochem. 157, 334-350 (2020).

40. Root, J., Merino, P., Nuckols, A., Johnson, M. \& Kukar, T. Lysosome dysfunction as a cause of neurodegenerative diseases: lessons from frontotemporal dementia and amyotrophic lateral sclerosis. Neurobiol. Dis. 154, 105360 (2021)

41. Amick, J., Tharkeshwar, A. K., Amaya, C. \& Ferguson, S. M. WDR41 supports lysosomal response to changes in amino acid availability. Mol. Biol. Cell 29, 2213-2227 (2018).
42. Amick, J., Tharkeshwar, A. K., Talaia, G. \& Ferguson, S. M. PQLC2 recruits the C9orf72 complex to lysosomes in response to cationic amino acid starvation. J. Cell Biol. 219, e201906076 (2020).

43. Talaia, G., Amick, J. \& Ferguson, S. M. Receptor-like role for PQLC2 amino acid transporter in the lysosomal sensing of cationic amino acids. Proc. Natl Acad. Sci. U. S. A. 118, e2014941118 (2021).

44. Zeeh, J. C., Antonny, B., Cherfils, J. \& Zeghouf, M. In vitro assays to characterize inhibitors of the activation of small $G$ proteins by their guanine nucleotide exchange factors. Methods Enzymol. 438, 41-56 (2008).

45. Zheng, S. Q. et al. MotionCor2: anisotropic correction of beam-induced motion for improved cryo-electron microscopy. Nat. Methods 14, 331-332 (2017).

46. Rohou, A. \& Grigorieff, N. CTFFIND4: fast and accurate defocus estimation from electron micrographs. J. Struct. Biol. 192, 216-221 (2015).

47. Zivanov, J. et al. New tools for automated high-resolution cryo-EM structure determination in RELION-3. Elife 7, e42166 (2018)

48. Punjani, A., Rubinstein, J. L., Fleet, D. J. \& Brubaker, M. A. cryoSPARC: algorithms for rapid unsupervised cryo-EM structure determination. Nat. Methods 14, 290-296 (2017).

49. Sanchez-Garcia, R. et al. DeepEMhancer: a deep learning solution for cryo-EM volume post-processing. BioRxiv https://doi.org/10.1101/2020.06.12.148296 (2020).

50. Zhang, K. Gctf: real-time CTF determination and correction. J. Struct. Biol. 193, 1-12 (2016).

51. Tan, Y. Z. et al. Addressing preferred specimen orientation in single-particle cryo-EM through tilting. Nat. Methods 14, 793-796 (2017).

52. Goddard, T. D. et al. UCSF ChimeraX: meeting modern challenges in visualization and analysis. Protein Sci.: Publ. Protein Soc. 27, 14-25 (2018).

53. Hintze, B. J., Lewis, S. M., Richardson, J. S. \& Richardson, D. C. Molprobity's ultimate rotamer-library distributions for model validation. Proteins $\mathbf{8 4}$, 1177-1189 (2016)

\section{Acknowledgements}

We thank Roberto Zoncu and Aaron Joiner for discussions, and Richard Hooy for workstation support. This work was supported by Department of Defense Peer Reviewed Medical Research Program Discovery Award W81XWH2010086 (J.H.H.), NIH R01 GM111730 (J.H.H.), a postdoctoral fellowship from the Association for Frontotemporal Degeneration (M.-Y.S.), and an EMBO Long-Term postdoctoral fellowship (S.A.F.).

\section{Author contributions}

M.-Y.S. and J.H.H. conceived the overall project, designed the study, and wrote the original draft. M.-Y.S. designed and carried out all the experiments. S.A.F. advised on experimentation and validated the model. J.R. and D.B.T. assisted in cryo-EM data collection. All authors contributed to reviewing and editing the manuscript.

\section{Competing interests}

J.H.H. is a scientific founder of Casma Therapeutics. The other authors declare no competing interests.

\section{Additional information}

Supplementary information The online version contains supplementary material available at https://doi.org/10.1038/s41467-021-24081-0.

Correspondence and requests for materials should be addressed to J.H.H.

Reprints and permission information is available at http://www.nature.com/reprints

Publisher's note Springer Nature remains neutral with regard to jurisdictional claims in published maps and institutional affiliations.

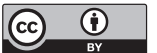

Open Access This article is licensed under a Creative Commons Attribution 4.0 International License, which permits use, sharing, adaptation, distribution and reproduction in any medium or format, as long as you give appropriate credit to the original author(s) and the source, provide a link to the Creative Commons license, and indicate if changes were made. The images or other third party material in this article are included in the article's Creative Commons license, unless indicated otherwise in a credit line to the material. If material is not included in the article's Creative Commons license and your intended use is not permitted by statutory regulation or exceeds the permitted use, you will need to obtain permission directly from the copyright holder. To view a copy of this license, visit http://creativecommons.org/ licenses/by/4.0/.

(C) The Author(s) 2021 